\title{
Production of transverse controllable laser density distribution in Fermilab/NICADD photoinjector*
}

\author{
Jianliang Li ${ }^{1,2, \#}$, Rodion Tikoplav ${ }^{1,2},{\text { Philippe } \operatorname{Piot}^{1}, \text { Adrian Melissinos }}^{2}$ \\ ${ }^{1}$ Fermi National Accelerator Laboratory, MS 306, PO Box 500, Batavia, IL 60510 \\ ${ }^{2}$ Department of Physics and Astronomy, University of Rochester, Rochester, NY 14627
}

\begin{abstract}
The Fermilab/NICADD photoinjector laboratory consists of a photoemission electron source based on an L band rfgun. The CsTe photocathode is illuminated by an ultrashort UV laser. The transport line from the laser to the photocathode was recently upgraded to allow imaging of an object plane located $\sim 20 \mathrm{~m}$ from the photocathode. This upgrade allows the generation of transverse laser distributions with controllable non-uniformity, yielding the production of an electron beam with various transverse densities patterns. Measuring the evolution of the artificial pattern on the electron bunch provides information that can be used to benchmark numerical simulations and investigate the impact of space charge. Preliminary data on these investigations are presented in the present paper.
\end{abstract}

\section{INTRUDUCTION}

Electron beams with high brightness are required by many applications, such as free-electron laser, electron cooling for high-energy colliders and heavy-ion ignited inertial fusion. This need can be well met by "photoinjector" [1], which is a microwave cavity-based electron gun driven by ultrashort high-intensity laser. However, one limiting mechanism of low emittance for photoinjector is the conversion of space-charge potential energy into kinetic energy (emittance). According to the theory, a non-uniform beam has higher field energy than the equivalent uniform beam, and the particle distribution will become uniform as the energy difference is converted into transverse kinetic energy and hence emittance increases. This limitation is present whenever an electron beam with non-uniformity propagates along the focusing beam line. A lot of research efforts have [2-5] been invested in the process of converting space-charge potential energy to emittance. Therefore, it is important to investigate this conversion by studying the evolution of the electron non-uniformity in the ways of both experiment and simulation.

The Fermilab/NICADD photoinjector laboratory (FNPL) consists of a photoemission electron source based on an L-band ( $\mathrm{f}=1.3 \mathrm{GHz})$ rf-gun [6]. The $\mathrm{Cs}_{2} \mathrm{Te}$ photocathode is illuminated by an ultra-short UV laser. The UV beam is obtained by quadrupling an amplified IR source seeded with a custom-made Nd:YLF laser

*Work supported by Universities Research Association Inc. under Contract No. DE-AC02-76CH00300 with US DOE, and by NICADD.

\# jianlian@fnal.gov oscillator. The UV beam is then transported to the photocathode via a $20 \mathrm{~m}$ long line immersed in vacuum.

\section{IMAGING RELAY SETUP}

It is a challenging job to relay the mask image in laser room onto photocathode, which is more than $20 \mathrm{~m}$ away. Due to the afraid of ionizing the air molecules and causing the refraction of UV beam, the entire beam transport line is vacuumed. For about ten years, only flat mirrors had been used to transport the UV beam. However this simple method poses some problems. For instance, the pointing instability of the beam in laser room will be much exaggerated after such a long transport distance and it is almost impossible to deliver any desired transverse beam shape.

To make the controllable pattern possible on photocathode at FNPL we recently upgraded the imaging system so that the image of the mask in laser room can be easily relayed to electron gun in cave.

The beam transport line is folded and there are only three turning corners, where the optics can be mounted in the boxes. Figure 1 is the schematic diagram of the transport beam line that is downgraded from the 3dimension real line.

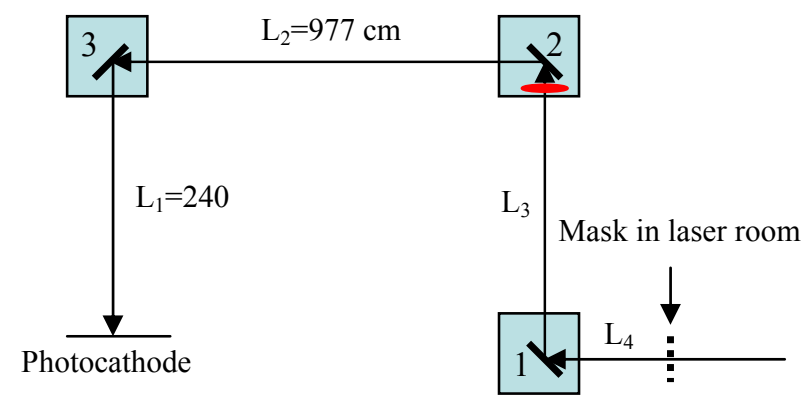

$$
\mathrm{L}_{3}+\mathrm{L}_{4}=870 \mathrm{~cm}
$$

Fig. 1. Schematic of he UV beam transport line.

We first tried to use a concaved spherical mirror to replace the flat mirror in box 2 . Soon it was realized that the focal lengths on two transverse dimensions are different because the concaved mirror needs also to reflect the beam for 90 degrees. This will result in different transverse image magnifications on photocathode, which means an elliptical image will be relayed from a round beam in laser room. A possible solution is parabola mirror, which is hard to seek and introduces difficulty on alignment.

To avoid the complicity and minimize the disturbance to the existing system, a standard singlet spherical plano- 
convex lens was inserted in front of the flat mirror in the turning box 2 . Ideally no deformation of the image is desired, so the unit magnification is sought. In reality, we are, however, limited by the fixed distances between the boxes and can only select the focal length of the lens. The lens equation requires that

$$
\frac{1}{S_{1}}+\frac{1}{S_{2}}=\frac{1}{f}
$$

where $\mathrm{S}_{1}=\mathrm{L}_{3}+\mathrm{L}_{4}=870 \mathrm{~cm}$ and $\mathrm{S}_{1}=\mathrm{L}_{1}+\mathrm{L}_{2}=1217 \mathrm{~cm}$. From equation (1), the focal length of the needed lens is determined to be $510 \mathrm{~cm}$ or so. The magnification is $\mathrm{S}_{1} / \mathrm{S}_{2} \approx 1.4$.

Before the installation of the lens, a test line was set up with HeNe laser to mimic the real line. The pictures on both mask and image planes were taken and shown in figure 2 . The ratio of the width of the line on the relayed image to that on the mask is 1.48 , close to the calculated magnification. The difference can be explained by the fact that the focal length for HeNe laser $(632.8 \mathrm{~nm})$ is slightly longer than that of the real UV beam with wavelength of $263 \mathrm{~nm}$.
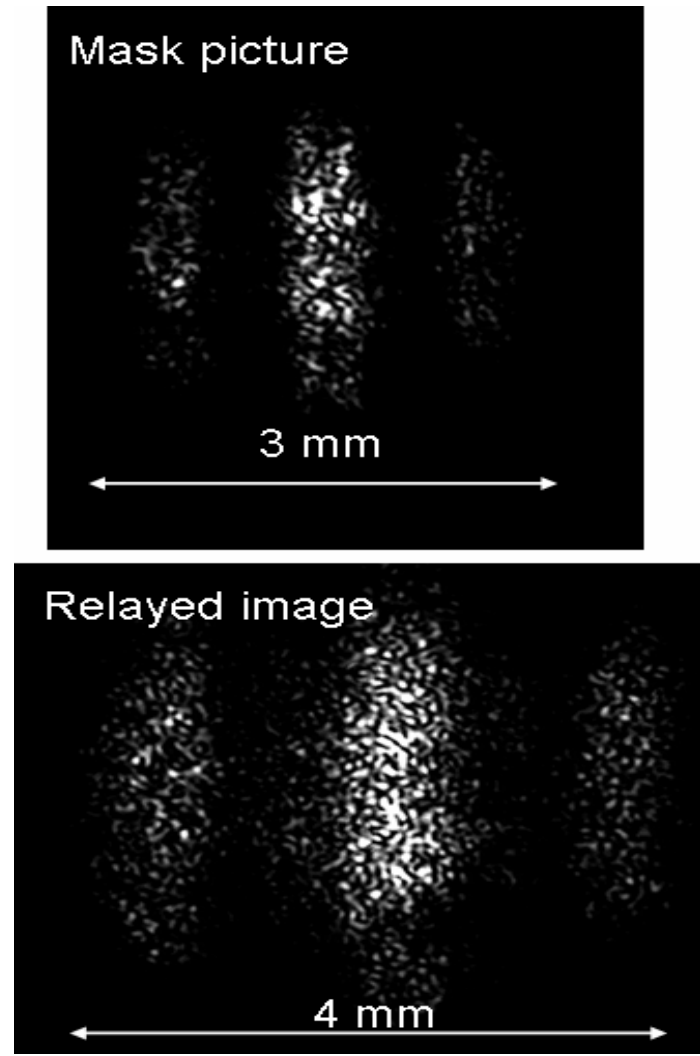

Fig. 2. Pictures were taken from mask in the test line with HeNe laser (above) and relayed image (below). The ratio of the width of the lines is close to design (see text).

\section{QUINCUNX EXPERIMENT}

\section{Experimental set-up}

Upon successful commissioning of our imaging beamline, we performed a preliminary experiment dedicated to generate and propagate an electron beam consisting of 5 beamlets organized as a quincunx. An aluminium mask was placed in the UV laser path at the location of the object plane and the resulting image observed on the virtual photocathode (a calibrated UVsensitive screen, located outside of the vacuum chamber, being a one-to-one optical image of the photocathode); see Fig. 3. The thereby photoemitted electron bunch exits the gun with an energy of approximately $4 \mathrm{MeV}$ and is further accelerated in a TESLA-type booster cavity operated at an average accelerating gradient of $12 \mathrm{MV} / \mathrm{m}$; the final energy is approximately $15.7 \mathrm{MeV}$. The beam transverse envelope was controlled only using the three solenoidal magnetic lenses that surround the rf-gun. The beam transverse density was then observed on a YAG screen located $4.6 \mathrm{~m}$ downstream of the photocathode, and recorded using a digital camera

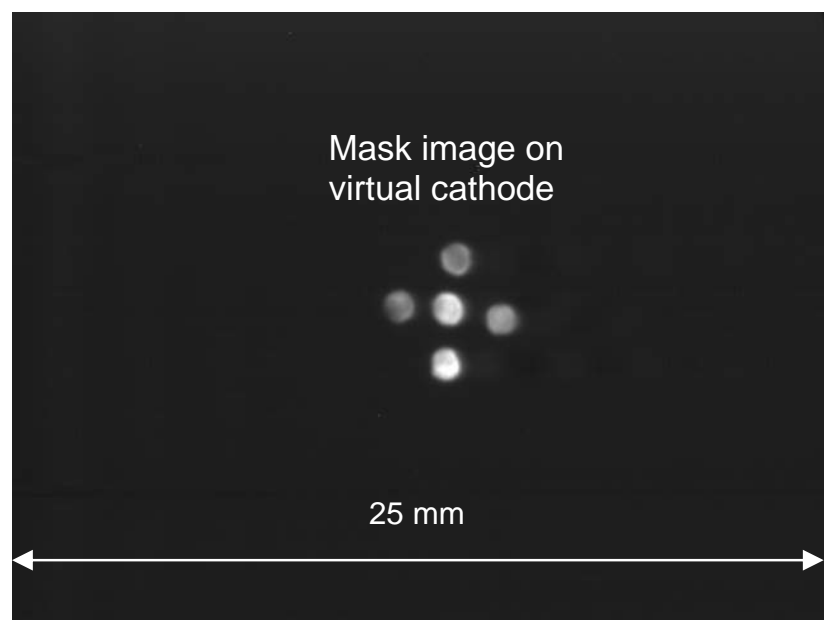

Fig. 3. Five beamlets image, taken from the virtual cathode in laser room, introduced artificial pattern on the initial electron beam.

\section{Experimental results and Simulations}

In the course of our experiment, the bunch charge was $\mathrm{Q}=440+/-100 \mathrm{pC}$, and the initial laser transverse density could not be made perfectly uniform (as shown in Fig. 3). The experiment consisted in observing the beam transverse density at the YAG screen location for different excitation currents of the solenoidal lens. The three lenses are operated with the same current for this experiment. The experiment is compared with simulation performed with the computer program Impact [7]. Impact incorporates a 3D space charge algorithm. Such an algorithm is mandatory for calculating the beam dynamics associated to beams with no cylindrical symmetry. In the present simulations, 200000 macro-particles were used to model the beam. The initial laser density (Fig. 3) is used to generate the input distribution for tracking into Impact. In the present case however the beamlets were assumed to be uniform with the same intensity (in reality this is not the case but the non-uniformity varies from shot-to-shot). For the tracking the rf-gun and TESLA cavity were set to reproduce the experimental conditions.

An example of a sequence of recorded pictures is presented in Fig. 4. As expected the electron beam retains 

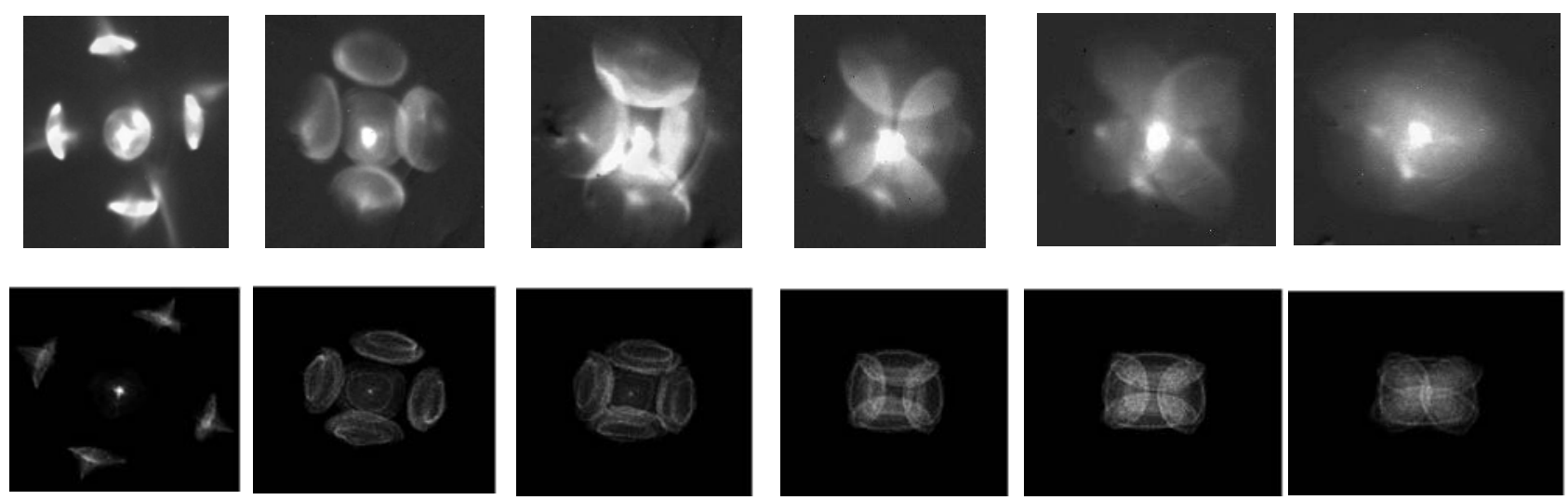

Fig. 4. Comparison of results from experiment (up row) and simulation (low row). The currents on primary solenoid are, from left to right, 237 A, 209 A, 199 A, 188 A, 182 A, and 173 A (the images are not to scale)

the quincunx pattern but the different beamlets undergoes different dynamics. The numerical simulations (also shown in Fig. 4) reproduce the general features of the experimental data. For instance at high solenoid current, the central beamlet consists of a charge concentration with diamond-shaped, while the other beamlet are oblate; these features is reproduced by the calculations. At lower current $<188$ A some of the features are not very well reproduced (for instance the bright core observed in the experiment does not appear in the simulations). We should emphasis that the experimental data are very preliminary: a certains number of parameters were not properly controlled (bunch charge from shot-to-shot and laser pulse length) and the on-going upgrade of our laser should fix these problems.

\section{SUMMARY}

As a part of the photocathode-drive laser upgrade for the FNPL, we have installed an imaging system that relay an object point in the laser room to the photocathode plane. Such a relaying optics has proven to ease the operation of a pulse stacker, and allows the introduction of shaped transverse spot size. The latter feature allows the generation of complex pattern enabling beam dynamics experiments (aimed to conversion of potential energy in emittance) and benchmarking of space-charge algorithm. A preliminary experiment was performed but a more thorough experiment will be done soon once we have finalized the upgrade of our laser system.

\section{References}

[1] X. J. Wang, in Proceeding of the PAC01, Chicago, IL, 2001, p. 81-85.

[2] J. D. Lawson, The physics of Charge Particle Beams (Oxford University Press, New Your, 1998).

[3] J. Struckmeier, J. Kalbunde, and M. Reiser, Part. Accel. 15, 47 (1984).

[4] I. Haber, D. Kehne, M. Reiser and H. Rudd, Physical Review A, Vo. 44, 5194 (1991).

[5] F. Zhou, I. Ben-Zvi, M. Babzien, X. Y. Chang, A. Doyuran, R. Malone, X. J. Wang and V. Yakimenko, Phys. Rev. Special Topics, Vol. 5, 094203-1 (2002).

[6] http://nicadd.niu.edu/fnpl

[7] J. Qiang et al., paper \#WPAP055, PAC 2005. 